\title{
Opportunities for Technology in the Self-Management of Mental Health
}

Elizabeth L. Murnane

Information Science

Cornell University

elm236@cornell.edu

\section{Mark Matthews}

Information Science

Cornell University

mark.matthews@cornell.edu

Geri Gay

Communication,

Information Science

Cornell University

gkg1@cornell.edu

Permission to make digital or hard copies of part or all of this work for personal or classroom use is granted without fee provided that copies are not made or distributed for profit or commercial advantage and that copies bear this notice and the full citation on the first page. Copyrights for third-party components of this work must be honored. For all other uses, contact the Owner/Author.

Copyright is held by the owner/author(s).

MobileHCl '16 Adjunct, September 06-09, 2016, Florence, Italy

ACM 978-1-4503-4413-5/16/09.

http://dx.doi.org/10.1145/2957265.2965008

\begin{abstract}
Mental health is becoming an increasingly pressing healthcare issue on a worldwide level. Chronic mental health conditions such as bipolar disorder are some of the most challenging illnesses to treat and are associated with considerable negative consequences, both in terms of societal costs as well as individual patients' quality of life. Mobile and wearable devices, with their rising ownership levels and sensing capabilities, have the potential to enable more personalized and broadly deployable forms of condition monitoring, symptom detection, and timely intervention. In this workshop paper, we overview our research into the lived experiences and self-management practices of individuals with bipolar disorder, the resultant implications for designing technology-based solutions, and the steps we have taken towards development of such assessment and intervention oriented tools. Importantly, we surface tensions between the opportunities of technology and the potential risks associated with their usage in the context of mental health.
\end{abstract}

\section{Author Keywords}

Mental Health; Bipolar Disorder; mHealth; Mobile Sensing; Self-Assessment; Intervention

\section{ACM Classification Keywords}

J.3 [Life and Medical Sciences]: Health 


\section{Introduction}

Mental health issues affect a significant portion of the world's population and can result in debilitating and life-threatening outcomes. Globally, about 450 million people suffer from mental illnesses, and neuropsychiatric disorders make up 4 of the 6 leading causes of years lived with disability. Recognized as one of the 10 most debilitating illnesses worldwide, bipolar disorder (BD) specifically affects approximately 60 million people. Medications and therapeutic techniques can help reduce BD symptoms, but it is still associated with an alarming suicide rate that is estimated to be 20 times higher than in the general population.

$\mathrm{BD}$ is characterized by episodes of mania and depression that are separated by periods of normal mood. Manic symptoms include elevated or irritable mood, hyperactivity, impulsivity, and sleep loss, while depressive symptoms include inactivity, fatigue, and social withdrawal. BD is chronic, and there is no cure. People diagnosed with BD expect to manage their condition for the rest of their lives.

Tracking and stabilizing behavior is considered a particularly effective non-pharmacologic treatment for diminishing symptoms of BD. In this paper, we overview our research aimed at exploring opportunities for BD-management technologies that better support patients' expressed needs, are more condition-tailored than generic tools, and can be more broadly deployed than traditional treatments.

\section{Self-Monitoring Practices}

Through both large scale surveys $(\mathrm{N}>500)$ [5] and small scale qualitative interviews $(\mathrm{N}=10)$ [4], we have identified ways in which individuals manage their $B D$, benefits and limitations they perceive in their current tracking approaches, and attitudes towards technology-based forms of monitoring. For instance, participants' modalities of tracking commonly included paper-based (e.g., journals, calendars, sticky notes) as well as digital tools (e.g., apps, wearables, spreadsheets), which were used to record a range of indicators, with mood, sleep, finances, exercise, and sociability being among the most common. We also found that these self-tracking practices could vary and evolve over time, often in parallel with an individual's phase of illness.

The majority of participants in our studies expressed that this self-monitoring is beneficial in managing their $\mathrm{BD}$, for example to facilitate interactions with clinicians or support discovery of personal patterns, symptom triggers, and effective recovery strategies. Notably, our studies suggest that the self-awareness and opportunities for introspection afforded by self-tracking can also foster self-compassion and acceptance and that the ability to take a more direct role in managing one's own health provides an empowering sense of agency - which is seen as particularly meaningful by our participants since they commonly struggle with issues of identity or feeling out of control due to their BD.

Though clinician-initiated tracking of BD-relevant indicators like sleep-wake routines or social interactions traditionally involves paper-based self-report instruments, this format faces known limitations related to adherence, which is particularly challenged during manic and depressive periods; plus, patterns can be more difficult to identify through paper logs. Indeed, participants in our studies described advantages to technology-based self-monitoring tools that ease capture burdens, and mental health treatment protocols that involve technology are becoming increasingly acceptable and advocated by organizations such as the Institute of Medicine and National Council for Behavioral Health.

Still, our participants encountered shortcomings with existing technologies, including a lack of support in capturing BD-specific indicators (e.g., well-known manic or depres- 


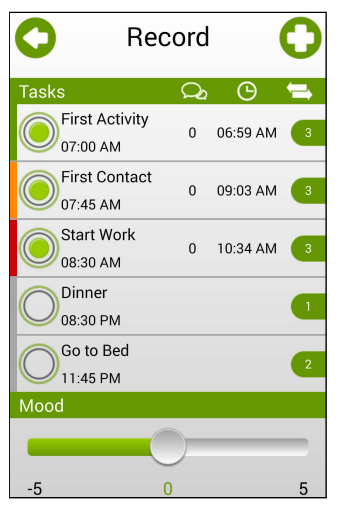

Figure 1: MoodRhythm's self-report screen

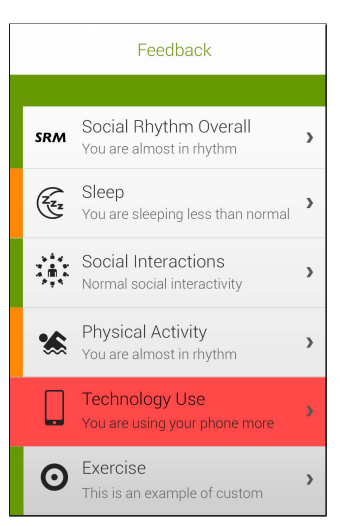

Figure 2: Real-time feedback

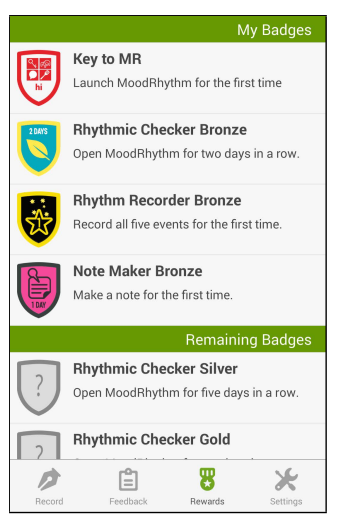

Figure 3: Badges rewarding adherence to self-monitoring sive prodromes, mixed moods, etc.) at a level of granularity sufficient for BD management (e.g., reporting multiple moods daily) as well as problems with the usability of interfaces that make them too cumbersome to use while experiencing symptoms. In light of such needs and to address such limitations, another line of our research has focused on developing a novel smartphone based platform for selfassessment, tailored feedback, and preemptive intervention for use in the management of bipolar disorder.

\section{Developing a Smartphone BD-Management Tool}

Our tool, "MoodRhythm" [3], is a patient-facing, cross-platform mobile app built on the Open mHealth Architecture and developed as part of a participatory design process involving clinicians, professional psychological researchers, and most importantly individuals with BD. Firstly, as illustrated in Figure 1, MoodRhythm helps patients track the 5 main behaviors used in the standard version of the Social Rhythm Metric (SRM), a paper-based daily measure of social routines that has been clinically validated to improve symptoms as a therapeutic self-monitoring instrument. Users can also add custom activities, set and track daily routine-related targets, and record notes. The app uses color indicators to provide glanceable feedback for the current and past days about how well an individual is hitting behavioral targets and maintaining routine regularity. It also provides weekly feedback using color as well as natural language summaries, as seen in Figure 2; and MoodRhythm rewards adherence to selfreporting with a variety of badges, as seen in Figure 3.

During a 4 week clinical pilot of MoodRhythm with 7 individuals with $\mathrm{BD}$ [1], the app received very high usability scores from participants, who particularly appreciated the convenience of recording activities with the smartphone version as well as its ability to provide feedback in real time. While these reactions are encouraging, an electronic ver- sion of the SRM still faces many of the same limitations as the paper form - the burden of remembering to track is still upon the user, and for individuals with BD, the reliability of self-assessment (especially during mania) is questionable for any self-report based instrument. Many of our participants did express a desire to have tools further relieve selfmanagement difficulties - for instance by proactively providing reminders to self-track or even passively monitoring behaviors without any explicit user input required.

Social rhythms and other BD-relevant parameters are particularly suitable candidates for automatic detection since they are objectively observable and do not require patients to actively reflect on an internal state. Further, smartphone sensing capabilities are well aligned with key BD indicators - namely, sleep-wake behaviors, activity levels, and social interaction. Based on criteria regarding cognitive and behavioral manifestations of manic and depressive episodes as well as our participants' own descriptions about ways in which their technology-mediated activities vary with symptoms, we can see how indicators of symptom onset could be passively collected using commonly available smartphone sensors or usage logging, as seen in Figure 4.

Pursuing that idea, MoodRhythm continuously captures information from various phone sensors. Our pilot study used features like location, distance traveled, and conversation frequency to predict mood stability with high accuracy [1]. Related research has had similar success inferring mood for individuals with $\mathrm{BD}$ using sensor data from their phones [2]. Such findings suggest the feasibility of using mobile systems for symptom detection and prediction, which could drive the next stage of support: providing preemptive care or targeted interventions, for instance through feedback that cautions about the potential onset of an episode or suggests personalized coping strategies for recovery. 


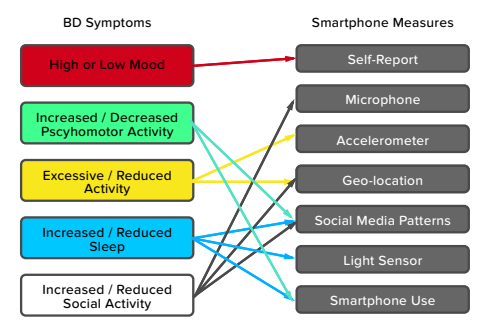

Figure 4: Bipolar symptoms and potential smartphone measures

\section{Significance to the Workshop}

Self-tracking of thoughts, behaviors, and emotions is an important resource for the self-management of a range of health conditions. In our work, we draw attention to selftracking as a means of managing mental health and focus specifically on the chronic condition of bipolar disorder. Our research contributes (1) an increased understanding of how individuals with BD manage their condition including via technology, (2) novel smartphone based solutions that relieve currently faced challenges with these approaches, and (3) preliminary mobile sensing techniques to passively detect and predict episodes. Moving forward, we are taking steps to close the loop of condition-management for BD by further expanding these tools to provide in-situ feedback that can help patients recover from a current episode or sustain behaviors to prevent future ones.

Developing self-management technology for the area of mental health also particularly helps to highlight a number of design tensions, given that in this context, self-tracking brings both therapeutic benefit and psychological risk. For instance, providing feedback about negative emotional and behavioral patterns could itself trigger or exacerbate symptoms; technology usage can have an agitating effect for individuals with $\mathrm{BD}$, which must be reconciled with the necessity of adherence; and while automated behavioral sensing can improve user burden and data fidelity, it can also elude the agency and empowerment tied to more explicit forms of self-monitoring. It is important for researchers and practitioners intending to develop or apply such technology to consider how to navigate these challenges as the community continues exploring effective and contextuallyappropriate strategies for health self-management.

\section{Acknowledgements}

Elizabeth Murnane is supported by the National Science Foundation Graduate Research Fellowship under Grant DGE 1144153

\section{REFERENCES}

1. Saeed Abdullah, Mark Matthews, Ellen Frank, Gavin Doherty, Geri Gay, and Tanzeem Choudhury. 2016. Automatic detection of social rhythms in bipolar disorder. Journal of the American Medical Informatics Association 23, 3 (2016), 538-543.

2. Mads Frost, Afsaneh Doryab, Maria Faurholt-Jepsen, Lars Vedel Kessing, and Jakob E Bardram. 2013. Supporting disease insight through data analysis: refinements of the monarca self-assessment system. In Proceedings of the ACM international joint conference on Pervasive and ubiquitous computing. 133-142.

3. Mark Matthews, Saeed Abdullah, Elizabeth L Murnane, Stephen Voida, Tanzeem Choudhury, Geri Gay, and Ellen Frank. 2016. Development and evaluation of a smartphone-based measure of social rhythms for bipolar disorder. Assessment (2016). (To appear).

4. Mark Matthews, Elizabeth L Murnane, and Jaime Snyder. 2017. Quantifying the Changeable Self: The role of self-tracking in coming to terms with and managing bipolar disorder. Human-Computer Interaction (2017). (Under review)

5. Elizabeth L Murnane, Dan Cosley, Pamara Chang, Shion Guha, Ellen Frank, Geri Gay, and Mark Matthews. 2016. Self-monitoring practices, attitudes, and needs of individuals with bipolar disorder: implications for the design of technologies to manage mental health. Journal of the American Medical Informatics Association 23, 3 (2016), 477-484. 